This is a post-peer-review, pre-copyedit version of an article published in European Journal of Clinical Nutrition.

The final authenticated version is available online at https://doi.org/10.1038/s41430-020-00820-2 


\title{
Dietetic management of obesity in Europe: Gaps in current practice
}

Running title: Gaps in dietetic management of Obesity in Europe

Vlassopoulos Antonis a, a,f, Govers Elisabeth ${ }^{\mathrm{c}, \mathrm{f}, \mathrm{g}}$, Mulrooney Hilda ${ }^{\mathrm{d}, \mathrm{f}}$, Androutsos Odysseas ${ }^{\mathrm{e}, \mathrm{f}}$, Hassapidou Maria ${ }^{b, f, g}$

\section{Author affiliations}

a Department of Food Science \& Human Nutrition, Agricultural University of Athens, Greece

${ }^{\mathrm{b}}$ Department of Nutritional Sciences and Dietetics, International Hellenic University, Thessaloniki, Greece,

${ }^{\mathrm{c}}$ Dutch Knowledge Centre for Dietitians on Obesity, Netherlands

${ }^{d}$ Kingston University, London \& Obesity Group of the British Dietetic Association, UK

${ }^{\text {e }}$ Department of Nutrition \& Dietetics, University of Thessaly, Trikala, Greece

${ }^{f}$ European Specialist Dietetic Network on Obesity, European Federation of the Associations of Dietitians (EFAD)

${ }^{g}$ Nutrition Working Group, European Association for the Study of Obesity (EASO)

\section{Corresponding Author}

Prof. Maria Hassapidou

Department of Nutritional Sciences and Dietetics

International Hellenic University

PO Box: 141

Sindos 57400

Thessaloniki, Greece

mnhas@nutr.teithe.gr

Keywords: Dietary management, Obesity, dietary treatment, guidelines, recommendations

\begin{abstract}
Despite substantial attention to dietary interventions on the management of obesity, there are no consensus guidelines for dietetic management of obesity in Europe. Two surveys among European dietitians have demonstrated inconsistencies in the approaches recommended within national obesity treatment guidelines. Only a small number of the guidelines include concrete actionable targets for recommended energy deficit, weight loss and weight loss maintenance. On the other hand, dietitians frequently use 5-15\% weight loss as their intervention outcome. However, they fail to monitor changes in body composition beyond weight status and to successfully monitor and prevent weight regain. Europe wide guidelines on the dietary treatment of obesity is an overdue requirement for consistent dietetic practice.
\end{abstract}




\section{Introduction}

Since 2007, the EU Platform for Action on Diet, Physical Activity \& Health has been the forum to discuss strategies (reformulation, fiscal measures, on-pack labelling) that would have the capacity to reverse the increase in obesity prevalence in Europe ${ }^{1-8}$. Within this, European Federation of the Associations of Dietitians (EFAD) in collaboration with European Association for the Study of Obesity (EASO) has committed to address the current gap of activities on the dietetic management of established obesity. The current survey was designed to scope existing national guidelines specifically targeted on the dietary management of obesity, and audit for the first time current dietetic practice in Europe as a first step towards an EU wide strategy on obesity treatment.

\section{Methods}

Two online surveys were conducted to collect data on: i) existing dietetic guidelines for the management of obesity across Europe and ii) the current practice of dietitians treating patients living with obesity.

For both surveys, dietitians from 25 countries, all EFAD members and EASO members were contacted. Questionnaire dissemination was carried through the National Dietetic Associations to Obesity Specialist and not through the investigators, hence no response rate can be calculated. For the first survey, dietitians were asked to provide an English translation of their national guidelines for the dietetic treatment of obesity. They were asked to highlight the proposed weight loss goals, specific dietary regimes recommended or contraindicated, the evidence base used to produce the guidelines and any potential adjunctive treatments.

The second survey included a short questionnaire distributed to dietitians across Europe. This collected data on educational levels, current occupational settings and numbers of patients treated on a monthly basis. It also collected data on the anthropometric tools used for diagnosis and monitoring of changes to body weight and composition, existing national or regional guidelines they used, dietetic plans most commonly prescribed, weight reduction goals and tools used to assess quality and effectiveness of weight loss intervention.

All questionnaires were content validated by at least two independent researchers. No ethical approval was necessary as all data collection was anonymised and no personal or sensitive information was collected. Descriptive statistics were carried out using the SPSS 16.1 statistical software.

\section{Results}

\section{National guidelines:}

In total, nine countries responded to the first survey (country response rate 9/25). Seven of those provided specific guidelines for the dietetic management of obesity (Belgium, Germany, Great Britain, Greece, Italy, Netherlands, and Sweden). Two countries (France and Spain) mentioned that no guidelines were available. Where guidelines were available, in over half, they were targeted specifically to dietetic management. All guidelines referred to the epidemiology and aetiology of obesity and proposed evidence-based treatment of obesity that included diet. All guidelines reported the level of evidence available to support each recommendation. The importance of exercise as part of the management of obesity was reported in all guidelines except one (Greece). The need for behavioural or psychological components to treatment was only identified in four of the guidelines and even less 
commonly mentioned were combined therapy, the use of obesity medication and bariatric surgery. Four countries considered weight maintenance as part of the dietetic treatment of obesity.

Regarding the dietetic intervention itself, there was little agreement (see Table 1). The reduction of total fat intake, avoidance of sweet drinks, including juices and the manipulation of the glycaemic index were the most common dietetic interventions mentioned with the Netherlands and Sweden mentioning all of these. The provision of objective and measurable goals for energy deficit was specified by three countries, but with differing proposed targets (data not shown). Similarly, four countries provided targets for successful weight loss. Specifically, the countries proposing a 500-600 kcal daily energy deficit (Great Britain, Netherlands) also proposed 5-15\% weight loss, while Germany which proposed a larger deficit (800 kcal) proposed a minimum of $5-10 \%$ weight loss dependent on baseline BMI class (BMI 25 $35 \mathrm{~kg} / \mathrm{m}^{2}$ and $\mathrm{BMI}>35 \mathrm{~kg} / \mathrm{m}^{2}$ respectively). Great Britain, Italy and the Netherlands included specific targets for weight loss maintenance at 2- and 5-years post intervention. Germany mentioned the importance of weight loss maintenance but did not provide a specific goal.

\section{Audit of dietetic practice:}

A total of 229 dietitians from 22 countries participated. Of these, 185 (81\%) participants complete the totality of the questionnaire and were eligible for analysis. Participants worked in private practice or hospital settings ( $46 \%$ and $45 \%$, respectively) with a small number $(9 \%)$ working in other settings (bariatric surgery, endocrinology, rehabilitation, public health). Participants were highly educated with $38.2 \%$ of dietitians having at least one postgraduate degree. Specialist training in obesity management was reported by $65 \%$ of participants.

Although almost everyone reported measuring height, weight and BMI (90.1\%), additional anthropometry (waist circumference measurement or body composition by bioelectric impedance) was only used by $\sim 64 \%$ of the dietitians. The majority (76.7\%) reported following national guidelines for the treatment of obesity and $84 \%$ identified the guidelines used in their practice as multi-disciplinary. Simultaneously half of the dietitians reported having their own working guidelines.

The most common dietary intervention was based on the patient's dietary assessment, followed by the prescription of a Mediterranean type diet with more restrictive dietary interventions (low fat, low carbohydrate and very low calorie) used less often. Although most participants agreed on a weight loss range of $5-15 \%$, there was little agreement on the actual target. Other qualitative indices such as quality of life and improvement of comorbidities were also used. A small proportion considered weight loss maintenance as a treatment outcome although the majority considered an intervention successful if it stimulated long-term adherence (70.8\%). Improving body composition and gaining control over comorbidities were also considered as important markers of intervention success (53.5\%).

\section{Discussion \& Conclusion}

This is one of the few studies to collect data directly from dietitians specialized in obesity management, throughout Europe and compare their practice to existing guidelines. Previous research has shown that dietetic management is commonly mentioned as part of the multidisciplinary approach to treat obesity ${ }^{9}$ however based on our data the provision of guidelines targeted specifically to dietitians is limited. The combination of these two surveys, highlight that dietetic practice closely follows the guidelines issued and that the lack of provision of quantifiable targets is mirrored in real-life situations. According to the dietitians' survey, SMART (Specific, Measurable, Attainable, Relevant, Time-based) weight loss targets are widely employed in clinical practice however these targets may be decided based on previous knowledge/experience or borrowed by guidelines issued by other countries. In the case of weight-loss 
maintenance the disregard of the subject in the guidelines is directly translated in a similar dietetic practice and the same can be said about the use of multiple indicators for body composition assessment. The unorthodox data collection methodology that employed dietitians as the main source of data about guidelines instead of an extensive literature search could be considered the main limitation of this study. However, these two surveys were designed to provide a picture of the real-life conditions of dietary management of obesity. By conducting research directly through the end-users of the guidelines it was possible to include data from guidelines published only in the local language and increase the representativity of the results, as well as draw direct comparisons between guidance and practice. Through this approach, this survey is capable to identify the need for a more unified approach in the dietetic management of obesity in Europe. The provision of guidelines at a central level, applicable to all European countries would vastly resolve local discrepancies and will more effectively guide dietetic practice. What is more, a Europewide set of guidelines would diminish the need for local guidelines and replication of activities by multiple committees, leading to substantial improvements to resource management.

\section{Conflicts of Interest}

The authors declare no conflict of interest.

\section{Funding Source}

This study received no funding.

\section{Author contributions}

$A V$ and $H M$ wrote the first draft of the manuscript, $A V$ and $O A$ carried out the data analysis, EG carried out data collection, EG and $\mathrm{MH}$ conceived the study, all authors reviewed and contributed to the final manuscript.

\section{References}

1 Blundell JE, Baker JL, Boyland E, Blaak E, Charzewska J, De Henauw S et al. Variations in the Prevalence of Obesity among European Countries, and a Consideration of Possible Causes. Obes Facts 2017; 10: 25-37.

2 Ansell C, Miura S. Can the power of platforms be harnessed for governance? Public Adm 2020; 98: 261-276.

3 Halicka E, Rejman K, Kaczorowska J. Changes in EU Food Supply and the Activities of the European Platform for Action on Diet, Physical Activity and Health. Probl World Agric / Probl Rol Światowego 2018; 18: 175-182.

4 Belc N, Smeu I, Macri A, Vallauri D, Flynn K. Reformulating foods to meet current scientific knowledge about salt, sugar and fats. Trends Food Sci. Technol. 2019; 84: 25-28.

5 Monteiro CA, Moubarac JC, Levy RB, Canella DS, Da Costa Louzada ML, Cannon G. Household availability of ultra-processed foods and obesity in nineteen European countries. Public Health 
Nutr 2018; 21: 18-26.

6 WHO Regional Office for Europe. Better food and nutrition in Europe: Progress report. 2018http://www.euro.who.int/en/health-topics/diseaseprevention/nutrition/publications/2018/better-food-and-nutrition-in-europe-progress-report2018.

7 WHO Regional Office for Europe. Action Plan for the Prevention and Control of Noncommunicable Diseases in the WHO European Region 2016-2025. 2017.

8 Loring B, Robertson A. Obesity and inequities: Guidance for addressing inequities in overweight and obesity. World Heal Organ Eur 2014; : 1-6.

9 Semlitsch T, Stigler FL, Jeitler K, Horvath K, Siebenhofer A. Management of overweight and obesity in primary care-A systematic overview of international evidence-based guidelines. Obes. Rev. 2019; 20: 1218-1230.

Table 1 Analysis of the dietetic management of obesity national guidelines

Figure 1 Survey results on the a) treatment outcomes considered by dietitians when designing a dietary intervention for obesity management, b) diets commonly prescribed, c) anthropometry assessment methods used and d) criteria for the assessment of success in weight loss intervention. 